Original Contribution

\title{
COMPARATIVE ANALYSIS OF THE ENERGIES OF NEEDLE BAR MECHANISMS OF THE SEWING MACHINES
}

\author{
Bl. Paleva-Kadiyska* \\ Department "Mechanical Engineering and Technologies", Faculty of Engineering, \\ South-West University "Neofit Rilski”, Blagoevgrad, Bulgaria
}

\begin{abstract}
Introduction: Sewing machines a set of mechanisms that work together, of which the most dynamic mechanism is the one that drives the needle. Stitch quality, the stitch strength and the productivity of the sewing machine largely depend on the work of the needle bar mechanism. The main task during the study dynamics of the sewing machine mechanisms is to determine the energy balance. Purpose: The aim is to determine the total energy consumed by the mechanisms per one full rotation of the main shaft of each machine; the share of energy consumed by each unit of the needle bar mechanism for one turnover; the ratio of the energy of each of the links of the mechanism both during the working motion and during the free motion of the needle. Materials and Methods: The object of the study is the energies of the needle bar mechanisms of the sewing machines Juki DDL5550 and Textima 8332. An energy distribution analysis was performed during the working process and the free stroke of the sewing needle. The energies of the mechanisms of the two sewing machines were compared. Results: Were calculated maximum energy values are as follows: of the rocker: for Juki DDL5530 $\mathrm{E}_{\mathrm{k}}=2,8051 \mathrm{~kg} \cdot \mathrm{m}^{2} / \mathrm{s}^{2}$ and for Textima $8332 \mathrm{Ek}=$ $1,3864 \mathrm{~kg} . \mathrm{m}^{2} / \mathrm{s}^{2}$; of the executive links - the needle bars: for Juki DDL5530 $\mathrm{E}_{\mathrm{k}}=5,9507 \mathrm{~kg} \cdot \mathrm{m}^{2} / \mathrm{s}^{2}$ and for Textima $8332 \mathrm{E}_{\mathrm{k}}=4,3696 \mathrm{~kg} \cdot \mathrm{m}^{2} / \mathrm{s}^{2}$; general energy of needle bar mechanism: for Juki DDL5530 $\mathrm{E}_{\mathrm{k}}=$ $8,7487 \mathrm{~kg} \cdot \mathrm{m}^{2} / \mathrm{s}^{2}$ and for Textima $8332 \mathrm{E}_{\mathrm{k}}=5,7416 \mathrm{~kg} \cdot \mathrm{m}^{2} / \mathrm{s}^{2}$. Conclusion: The comparative analysis reveals that the energy consumption of the needle bar mechanism of Juki DDL5530 is significantly higher. Consequently, the energy consumption indicator of the Textima 8332, which is directly driven without a clamp, is more efficient.
\end{abstract}

Key words: analysis, energy balance, working process of the needle.

\section{INTRODUCTION}

Despite the introduction of different methods of joining details into the clothing, the main method is still by a sewing thread. In order to increase the productivity and quality of the stitching process, a number of improvements have been made to the sewing machine (SM) designs in recent years, related to increasing productivity, improving service conditions and sewing process quality.

$\mathrm{SM}$ is a complex of the mechanisms that work together. These mechanisms make necessary executive links motions for realizing the technological processes. The most dynamic mechanism is the one that drives the needle. Stitch quality, the stitch strength, and the productivity of the SM largely depend on its work.

\footnotetext{
"Correspondence to: Blagoyka Paleva-Kadiyska, PhD; 7 Pobeda str., Blagoevgrad; Bulgaria; e-mail: paleva-kadiyska.bl@abv.bg
}

The optimization of the kinematic and dynamic parameters of the needle bar mechanisms (NBM) during their interaction with the other mechanisms in SM are tasks that provide new opportunities for improving the process of sewing (1).

While working, each mechanism, including an NBM, transfers mechanical energy from the source to the consumer. The main task during the study of their dynamics is to determine the energy balance: determine the total amount of consumed energy used to realize the given movement, and study the laws for its distribution (2). By applying the kinetic energy theorem, the law of mechanical energy conservation and based on virtual work, it is significantly easier to establish the dependencies between the kinematic (velocity, acceleration) and dynamic (forces, moments, mass characteristics) parameters of motion of the bodies 
and the systems of solid bodies. There is no necessity to study the differential law of motion. Therefore the energy distribution between the links of the mechanisms and its change during work is essential for the work of SM.

The purpose of the study is to conduct a comparative analysis of two NBM, which have constructive differences in the connection between the rocker and the needle bar, by:

- Determine the total energy consumed by the mechanism per one full rotation of the main shaft of each machine;

- The share of energy consumed by each unit of the NBM for one turnover;

- The ratio of the energy of each of the links of the mechanism both during the working motion and during the free motion of the needle.

\section{MATERIALS AND METHODS}

Depending on the technological operation, the needle in the SM can perform different movements. The most widespread application in practice is the universal sewing machines with lock stitch class-301 in which the needle performs a reciprocating movement in a vertical direction. The task of the NBM is to transform the rotary motion of the main shaft into a reciprocation of the needle bar. In a SM, where the main shaft is located at the top of the machine, the conversion is performed by a slider-crank mechanism with crank 1 , rocker 2 and slider 3 - Figure 1.

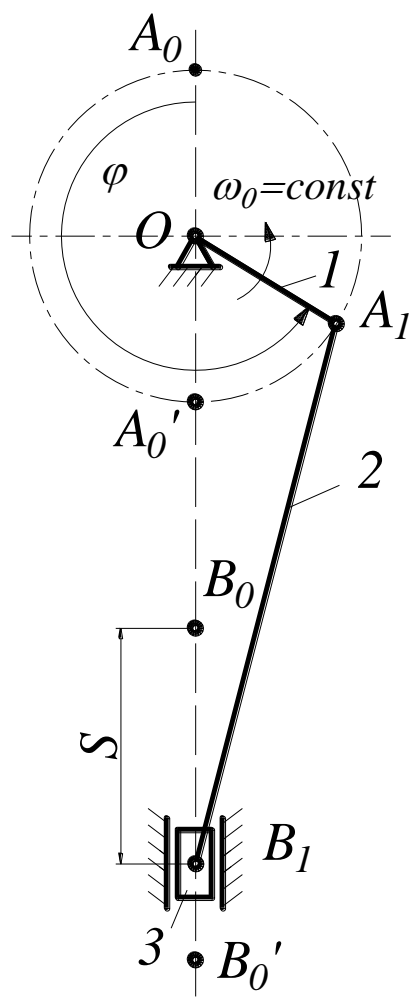

Figure 1. Kinematic scheme of axial slider-crank mechanisms

The study was conducted for the NBM of SM Juki DDL5530 and Textima 8332. Both mechanisms are axial slider-crank mechanisms. The path of movement of the needle passes through the center of rotation $O$ of the crank 1 $O A$. These mechanisms are of simple construction, provide greater steadiness of movement and smaller inertial forces than offset slider-crank mechanisms (non-axial) ones, and are therefore mainly used in modern highspeed sewing machines (3).
The two mechanisms are different in construction of the connection between the rocker 2 and the slider 3 (needle bar). In Textima 8332 it is direct - with an axle between them, and at Juki DDL 5530 is indirect - with an axle located on an additional detail - a clamp. The clamp is fixed to the needle bar 3 . This is the more common case in SM.

In both machines for stabilizing the vertical move of the needle bar a slider 3 is used. 
The study did not take into account the potential energy generated by the center of gravity and the deformation of the links as it was negligible. The energy created in the mechanism fulfills the law of conservation of energy.
PALEVA-KADIYSKA BL.

NBM is a mechanical system of connected rigid bodies. The kinetic energy of such a system, according to König's theorem, is at every time a sum of the energies of its constructing links ( $n$ number):

$$
E_{k j}=\frac{1}{2} \sum_{i=1}^{n}\left(m_{i} \cdot V_{S_{i j}}^{2}+J_{i} \cdot \omega_{i j}^{2}\right)
$$

where:

$E_{k j}$ - the kinetic energy of the mechanism in this moment, $\mathrm{kg} . \mathrm{m}^{2} / \mathrm{s}^{2}$;

$m_{i}$ - the mass of the i-th link, $k g$;

$V_{S_{i j}}$ - the velocity of the center of gravity of the i-th link in the j-th moment, $\mathrm{m} / \mathrm{s}$;

$J_{i}$ - the moment of inertia of the i-th link, with respect to an axis passing through the mass' center of the link, for the general case of planar motion, $\mathrm{kg} . \mathrm{m}^{2}$; $\omega_{\mathrm{ij}}$ - the angular velocity of the i-th link, $s^{-1}$.

\section{RESULTS}

Data for masses, for the moments of inertia and for velocities of the links were taken from (1), where a study of the NBM of SM Juki DDL5550 and Textima 8332 was conducted. Citing on that data, the kinetic energy of each of the links and the total energy of the mechanisms for one full turn of the main shaft was calculated. This was performed for each of the two SM.

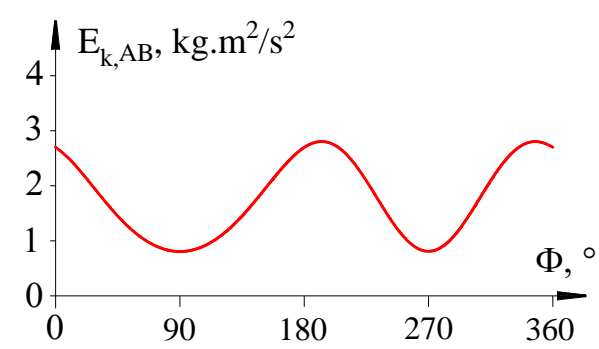

Figure 2. Kinetic energy of the rocker $A B$ of the needle bar mechanism of sewing machine Juki DDL5530

An energy examination revealed that there are peaks at the angles of rotation of the main machine shafts as follows: the first
The angular velocity $\omega_{0}$ of the rocker is a function of the angular velocity of the drive unit, $\omega_{\mathrm{i}}(\varphi)=\mathrm{f}\left(\omega_{0}\right)$, where: $\varphi$ - angle of rotation of the drive unit; $\mathrm{f}$ - is determined by the transfer functions of the mechanism.

The mass of the links, the moments of inertia and the position of their mass centers are constant quantities. The kinetic energy change function depends only on the angular velocity. To simplify the study is accepted $\omega_{0}=1$. Resisting force of the merged details did not taken into account. It is different for each case and depends on the type of the textile material. The results are presented in graphical form Figure 2 to Figure 7.

The kinematic analysis performed in (1) indicates that the working process (as shown in Figure 1) of the sewing needle in both machines starts at about $180^{\circ}$ and ends at about $360^{\circ}$. These are the two moments - the one in which the needle passes through the material and the second one the needle leaves the material.

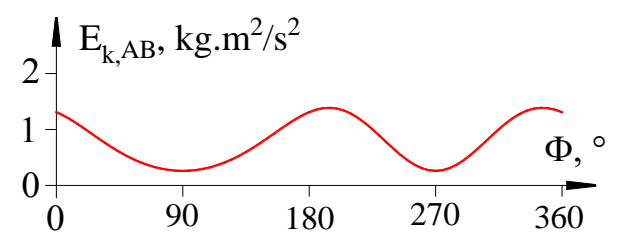

Figure 3. Kinetic energy of the rocker $A B$ of the needle bar mechanism of sewing machine Textima 8332

approximately $15^{\circ}-20^{\circ}$ after the start of the needle working process and the second $15^{\circ}-20^{\circ}$ before its end. 


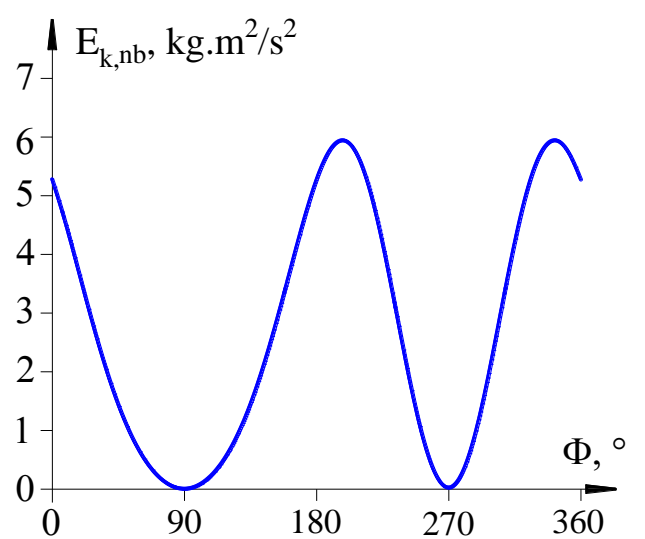

Figure 4. Kinetic energy of the needle bar of Juki DDL5530

The calculated maximum energy values are:

1) of the rockers:

- for Juki DDL5530 - at $195^{\circ}$ and at $345^{\circ}$

$E_{k, A B}=2,8051 \mathrm{~kg} . \mathrm{m}^{2} / \mathrm{s}^{2}$.

- for Textima 8332 - at $195^{\circ}$ and at $345^{\circ}$

$E_{k, A B}=1,3864 \mathrm{~kg} . \mathrm{m}^{2} / \mathrm{s}^{2}$.

2) of the executive links - the needle bars:

- for Juki DDL5530 - at $200^{\circ}$ and at $340^{\circ}$

$E_{k, n b}=5,9507 \mathrm{~kg} \cdot \mathrm{m}^{2} / \mathrm{s}^{2}$.

- for Textima 8332 - at $200^{\circ}$ and at $340^{\circ}$

$E_{k, n b}=4,3696 \mathrm{~kg} \cdot \mathrm{m}^{2} / \mathrm{s}^{2}$.

3) general energy of both NBMs:

- for Juki DDL5530 - at $195^{\circ}$ and at $345^{\circ}$

$E_{k}=8,7487 \mathrm{~kg} \cdot \mathrm{m}^{2} / \mathrm{s}^{2}$.

- for Textima 8332 - at $200^{\circ}$ and at $340^{\circ}$

$E_{k}=5,7416 \mathrm{~kg} \cdot \mathrm{m}^{2} / \mathrm{s}^{2}$.

The curve presents the magnitude of the kinetic energy at a given moment does not correspond to the obtained graph of the angular velocity $\omega$ of the reduced link. Extremes of $E=E(\varphi)$ on the NBMs' rockers have abscissas that do not coincide with the abscissa of the extremes of

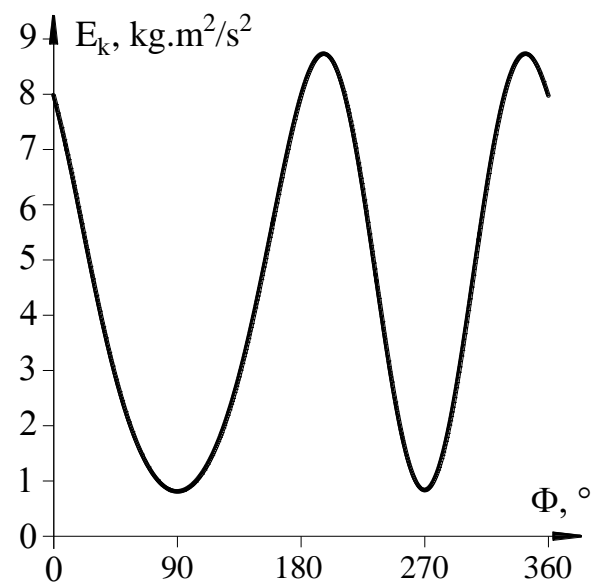

Figure 6. The kinetic energy of the needle bar mechanism of the Juki DDL5530

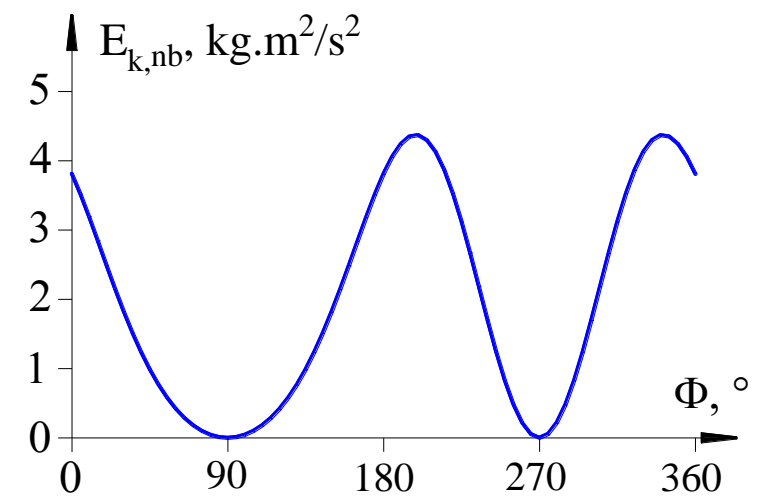

Figure 5. Kinetic energy of the needle bar of Textima 8332

$\omega=\omega(\varphi)$. This is because the kinetic energy is proportional to $\omega^{2}$ and to the reduced moment of inertia $J_{r}=J_{r}(\varphi)$ also, which is a function of $\varphi$.

The conducted study reveals that the needlebar's rocker of Juki DDL5530 maximum energies is greater (almost twice) than those of the energy of the rocker needle bar of Textima 8332. This is shown in Figure 2 and Figure 3.

The results of energy of the end link (Figure 4 and Figure 5) are similar. The calculations show that the energy of Juki DDL5530 IMU executive unit (needle bar) is greater than the executive unit (needle bar) of Textima 8332.

The analysis of the energy balance of the needle bar mechanism reveals that the main influence on the consumed energy and the distribution law of the consumed energy for both SM has the kinetic energy of the needle bar (end link).

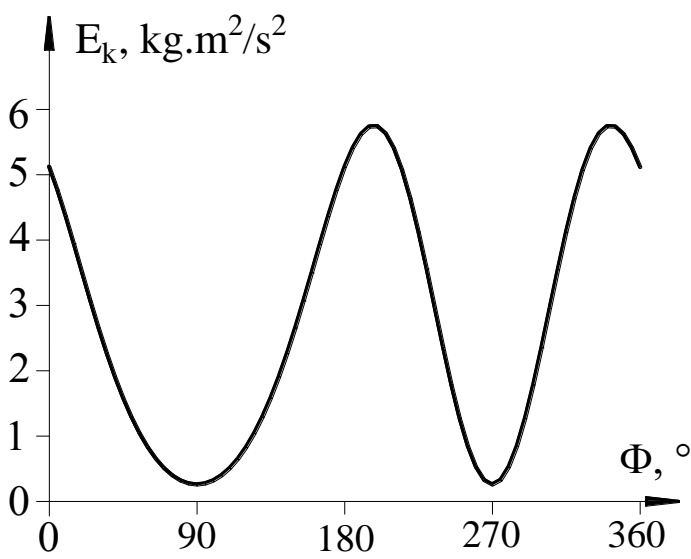

Figure 7. Kinetic energy of the driving needle bar mechanism of the Textima 8332 
The work done by the forces and moments acting on the units of the NBM for a fixed period of time can be determined by modifying
PALEVA-KADIYSKA BL.

the energy of the mechanism for the same fixed period. The energy model of the motion equation:

$$
\int_{0}^{2 \pi} J \omega d \omega=\int_{0}^{2 \pi} M(\varphi) d \varphi
$$

is integrated and know that the work done by the external forces acting on the system is:

$$
A=\int_{0}^{2 \pi} M(\varphi) d \varphi
$$

the work that has been done by the NBM and its links that build it is defined.

In the above formulas:

$A$ - the work performed by the external forces acting on the system in the 0 to $2 \pi$ angle of rotation $\varphi$ of the camshaft of the machine, kg. $\mathrm{m}^{2} / \mathrm{s}^{2}$

$J$ - reduced mass moment of inertia, $\mathrm{kg} . \mathrm{m}^{2}$;

$\omega-$ angular velocity of the camshaft, $s^{-1}$;

$M$ - reduced moment of active forces acting on mechanism,

An analysis of the work performed by NBM links of Juki DDL5530 and Textima 8332 per one rotation of the main shaft as well as during

Juki DDL5530

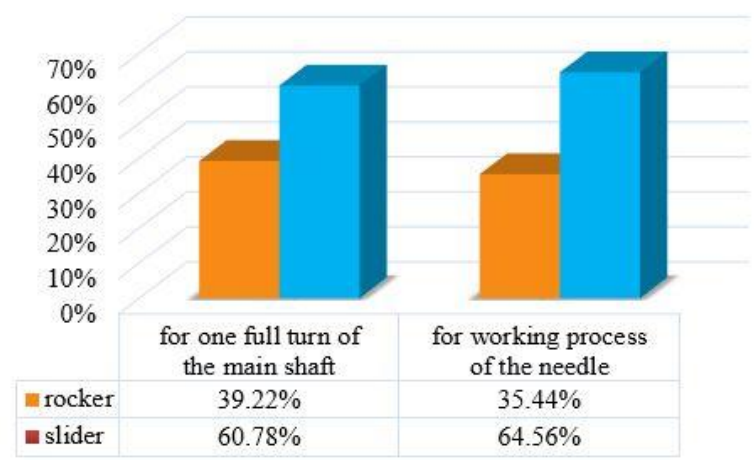

Figure 8. Comparison of energy consumed by the NBM links of Juki DDL 5530

The analysis results of the total energy spent by the NBM links of SM Textima 83323 are distributed as follows (Figure 9): about 1/3 (28.57\%) is spent by the mechanism's rocker and about $2 / 3$ by the slider (needle bar) $71.43 \%$. In the working process of the needle, some reduction of the energy consumed by the rocker (from $28.57 \%$ to $25.85 \%$ ) and an increase of the energy consumed by the slider (from $71.43 \%$ to $74.15 \%$ ) were recorded. working of the sewing needle is conducted with aim to be evaluated the consumed energy by these links.

The total energy distribution of the Juki DDL5530 needle bar is presented in Figure 8. In both periods (working process and free movement of the sewing needle), most of the energy of the mechanism is used by the executive link (needle bar). During the working process of the needle, there is a slight increase in the part of the energy consumed by the needle bar 3 (acc. to Figure1) (from $60.78 \%$ to $64.56 \%$ ), respectively the part of the energy consumed by the rocker 2 is reduced.

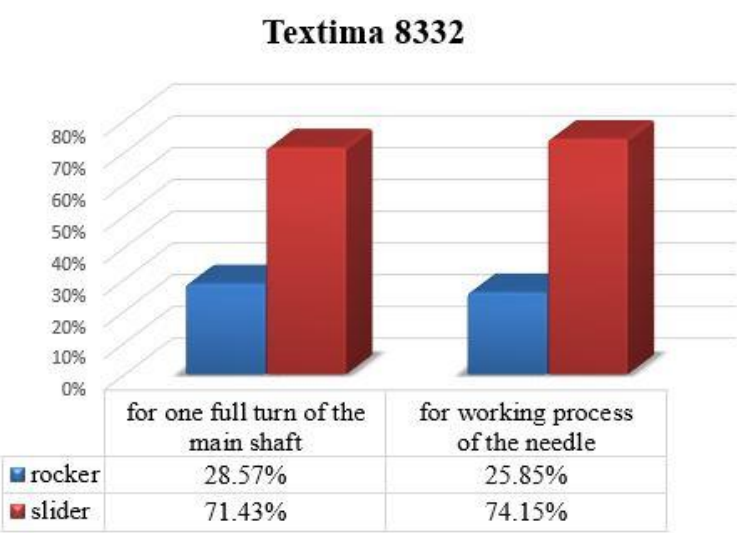

Figure 9. Comparison of energy consumed by the NBM links of Textima 8332

It is important to be determined how much of the energy of NBM links is spent during the needle's working process for clarification of the work of the NBM. In this study, are calculated the following ratios shown in Figure 10.

Over $60 \%(63.81 \%$ for Textima 8332 and $61.44 \%$ for Juki DDL 5530) of the total energy consumed by the NBM for a full turn of the main shaft of the machine is used to carry out its working process (from $180^{\circ}$ to $360^{\circ}$ ). 


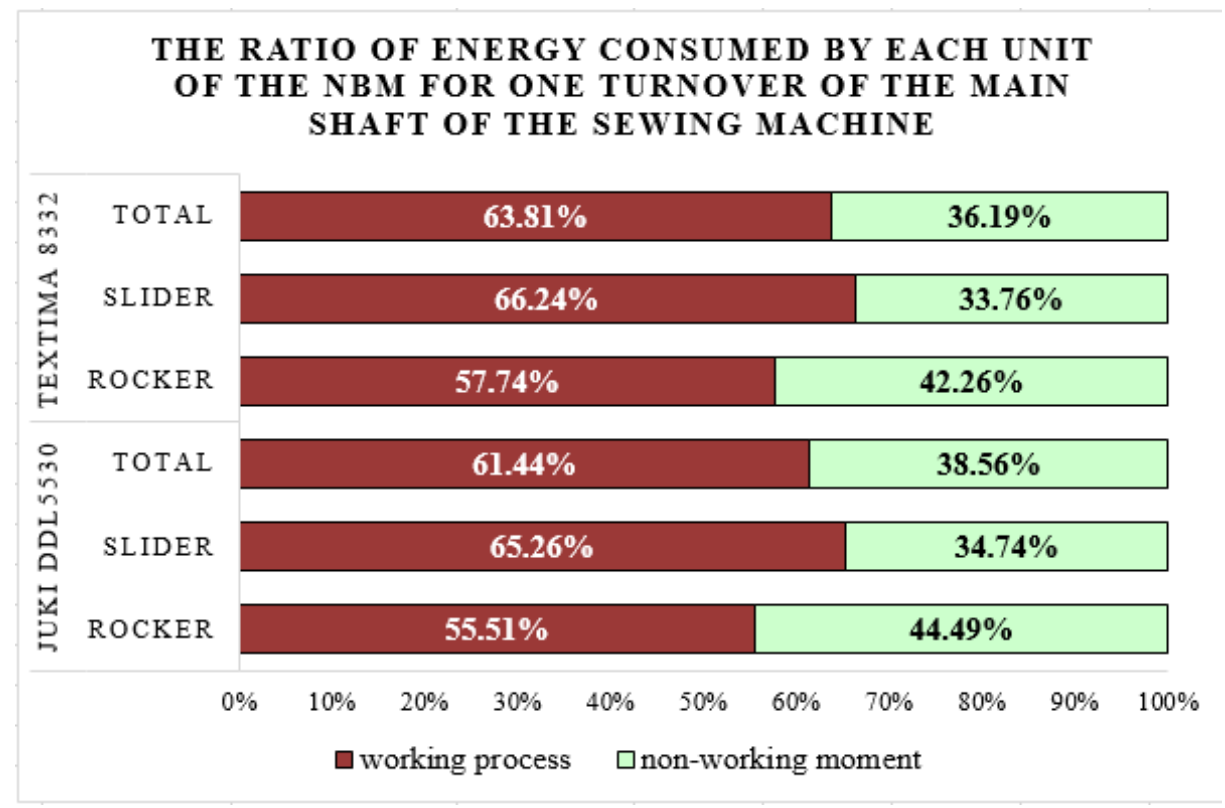

Figure 10. Comparison of energy consumed of NBM of Juki DDL 5530 and of Textima 8332 for a full turn of the main shaft

\section{CONCLUSION}

In pursuance of the stated objective, a study of the energy of the links and of the whole needle bar mechanism of both SM Juki DDL5530 and Textima 8332 was carried out. The results obtained from the comparative analysis provide the basis for the following conclusions:

- the change in kinetic energy of the links and needle bar mechanism is cyclical, smooth, without sharp changes;

- more significant influence on the extremums of the energy of the mechanism in both machines cause the peaks of the kinetic energies of the end links (the sliders);

- larger part of the total energy of the mechanism, for one full turn of the main shaft of the machine again belongs to the needle bar (slider);

- during needle's working process (from $180^{\circ}$ to $360^{\circ}$ ), the part of the energy of the links changes insignificantly (and needle bars again have a higher proportion).
- both maximums of the NBMs are during their working processes. This would lead to an increase in the energy balance peaks of the entire machine, as its load is further increased by the material resistance when the needle passes through the material;

The comparative analysis reveals that the energy consumption of the NBM of the Juki DDL5530 is significantly higher. This fact is due to the larger dimensions, masses and inertial moments of the links. Consequently, the energy consumption indicator of the Textima 8332, which is directly driven without a clamp, is more efficient.

\section{REFERENCES}

1. Balabanova, D. Research of needle bar mechanisms of the sewing machine. Dissertation for PhD, TU-Gabrovo, 2014 (in Bulgarian)

2. Minchev, N. and others. Theory of Mechanisms and Machines. Textbook, Technics, S., 1991 (in Bulgarian)

3. Trifonov K. Sewing machine and equipment. Textbook, Technics, S., 2004 (in Bulgarian) 